\title{
A Univariate, Bivariate and Multivariate Extensions for the Inverse Rayleigh Model with Properties and Applications to the Univariate Version
}

\author{
Wahhab Salim Mohammed \\ Department of Statistics, College of Administration and Economics, \\ Diyala University, Iraq.
}

Rania H. M. Abdelkhalek

Department of Statistics, Mathematics and Insurance, Benha University, Egypt.

\section{S. I. Ansari}

Department of Statistics, Faculty of Science, University of Tabuk, Tabuk, Kingdom of Saudi Arabia.

\begin{abstract}
A new univariate extension of the Inverse Rayleigh distribution is proposed and studied. Some of its fundamental statistical properties such as some stochastic properties, ordinary and incomplete moments, moments generating functions, residual life and reversed residual life functions, order statistics, quantile spread ordering, Rényi, Shannon and q-entropies are derived. A simple type Copula based construction via Morgenstern family and via Clayton copula is employed to derive many bivariate and multivariate extensions of the new model. We assessed the performance of the maximum likelihood estimators using a simulation study. The importance of the new model is shown via two applications to real data sets.
\end{abstract}

Keywords: Inverse Rayleigh Model; Morgenstern Family; Simulation; Clayton Copula; Modeling; Copula; Entropies; Wright Generalized Hypergeometric Function.

\section{Introduction and motivation}

A rv $W$ is said to have the Inverse Rayleigh (IR) distribution if its probability density function (PDF), cumulative distribution function (CDF) are given by

and

$$
h_{\lambda}(\omega)=2 \frac{\lambda^{2}}{\omega^{3}} \exp \left(-\lambda^{2} \omega^{-2}\right)
$$

$$
H_{\lambda}(\omega)=\exp \left(-\lambda^{2} \omega^{-2}\right)
$$

The cumulative distribution function (CDF) and probability density function (PDF) of the Generalized Odd Generalized Exponential G (GOGE-G) family are given, respectively, by

$$
\begin{gathered}
F_{\alpha, \beta}(\omega)=\left\{1-\exp \left[\frac{-H_{\Psi}(\omega)^{\alpha}}{1-H_{\Psi}(\omega)^{\alpha}}\right]\right\}^{\beta}, \\
f_{\alpha, \beta}(\omega)=\frac{\alpha \beta h_{\Psi}(\omega) H_{\Psi}(\omega)^{\alpha-1}}{\left[1-H_{\Psi}(\omega)^{\alpha}\right]^{2}} \exp \left[\frac{-H_{\Psi}(\omega)^{\alpha}}{1-H_{\Psi}(\omega)^{\alpha}}\right]\left\{1-\exp \left[\frac{-H_{\Psi}(\omega)^{\alpha}}{1-H_{\Psi}(\omega)^{\alpha}}\right]\right\}^{\beta-1},
\end{gathered}
$$


where $H_{\Psi}(\omega)$ is the baseline CDF depending on a parameter vector $\Psi$ and $h_{\Psi}(\omega)=$ $\frac{d}{d \omega} H_{\Psi}(\omega)$ is its corresponding PDF and $\alpha, \beta>0$ are two additional shape parameters. Using (3) and (2) the CDF of the GOGEIR can be derived as

$$
F_{\alpha, \beta, \lambda}(\omega)=\left(1-\exp \left\{\frac{-\exp \left(-\alpha \lambda^{2} \omega^{-2}\right)}{1-\exp \left(-\alpha \lambda^{2} \omega^{-2}\right)}\right\}\right)^{\beta},
$$

the corresponding PDF of (5) can be expressed as

$$
\begin{aligned}
& f_{\alpha, \beta, \lambda}(\omega)=\frac{2 \alpha \beta \lambda^{2} \omega^{-3}}{\left[1-\exp \left(-\alpha \lambda^{2} \omega^{-2}\right)\right]^{2}} \exp \left[\frac{-\exp \left(-\alpha \lambda^{2} \omega^{-2}\right)}{1-\exp \left(-\alpha \lambda^{2} \omega^{-2}\right)}\right] \\
& \times \exp \left(-\alpha \lambda^{2} \omega^{-2}\right)\left\{1-\exp \left[\frac{-\exp \left(-\alpha \lambda^{2} \omega^{-2}\right)}{1-\exp \left(-\alpha \lambda^{2} \omega^{-2}\right)}\right]\right\}^{\beta-1} .
\end{aligned}
$$

Henceforth, $W \sim \operatorname{GOGEIR}(\alpha, \beta, \lambda)$ denotes a random variable having density function (6). The hazard rate function (HRF) of $W$ can be derived using the well-known relationship

$$
f_{\alpha, \beta, \lambda}(\omega) /\left[1-F_{\alpha, \beta, \lambda}(\omega)\right]
$$

Plots of the GOGEIR HRF at some parameters value are presented in Figure 2 to show the flexibility of the new model. For simulating data from the GOGEIR model, if $u \sim u(0,1)$, then

$$
\omega_{u}=\lambda \sqrt{\left\{-\ln \left[\frac{-\log (1-\sqrt[\beta]{u})}{1-\log (1-\sqrt[\beta]{u})}\right]^{\frac{1}{\alpha}}\right\}^{-1}}
$$

has CDF (5). Now, we provide a useful representation for (6). Using the series expansion

$$
(1-c)^{\varphi}=\sum_{m=0}^{\infty} \frac{(-1)^{m} \Gamma(1+\varphi)}{m ! \Gamma(1+\varphi-m)} c^{m},
$$

which holds for $|c|<1$ and $\varphi>0$ real non-integer and using the power series, the PDF of the GOGEIR in (6) can be expressed as

$$
\begin{aligned}
& f(\omega)=\alpha \beta \lambda^{2} 2 \omega^{-3} \sum_{i, j_{1}=0}^{\infty} \frac{(-1)^{i+j_{1}}(i+1)^{j_{1}}}{j_{1} !}\left[1-\exp \left(-\alpha \lambda^{2} \omega^{-2}\right)\right]^{-\left(j_{1}+2\right)} \\
& \times\left(\begin{array}{c}
\beta-1 \\
i
\end{array}\right)\left[\exp \left(-\lambda^{2} \omega^{-2}\right)\right]^{\alpha\left(j_{1}+1\right)} .
\end{aligned}
$$

Using the series expansion again we arrive at

$$
f(\omega)=\sum_{j_{1}, j_{2}=0}^{\infty} v_{j_{1}, j_{2}} h_{\alpha\left(1+j_{1}+j_{2}\right)}(\omega)
$$

where $h_{\alpha\left(1+j_{1}+j_{2}\right)}(\omega)$ is the IR density with scale parameter $\lambda\left[\alpha\left(1+j_{1}+j_{2}\right)\right]^{\frac{1}{2}}$ and

$$
v_{j_{1}, j_{2}}=\alpha \beta \frac{(-1)^{j_{1}+j_{2}}}{j_{1} !\left[\alpha\left(1+j_{1}+j_{2}\right)\right]}\left(\begin{array}{c}
-\left(j_{1}+2\right) \\
j_{2}
\end{array}\right) \sum_{i=0}^{\infty}(-1)^{i}(i+1)^{j_{1}}\left(\begin{array}{c}
\beta-1 \\
i
\end{array}\right) \text {. }
$$


The CDF of the GOGEIR model can also be expressed as a mixture of the IR density CDFs. By integrating (8), we obtain the same mixture representation

$$
F(\omega)=\sum_{j_{1}, j_{2}=0}^{\infty} v_{j_{1}, j_{2}} H_{\alpha\left(1+j_{1}+j_{2}\right)}(\omega),
$$

where $H_{\alpha\left(1+j_{1}+j_{2}\right)}(\omega)$ is the CDF of the IR density with scale parameter $\lambda\left[\alpha\left(1+j_{1}+j_{2}\right)\right]^{\frac{1}{2}}$.

Some important extensions of the Fr model can be cited Nadarajah and Kotz (2003), Barreto-Souza et al. (2011), Mahmoud and Mandouh (2013), Krishna et.al. (2013), Yousof et el. (2015), Mead et al. (2016), Afify et al. (2016a), Weibull Fr Afify et al. (2016b), Yousof et al. (2016), Korkmaz et al. (2017), Yousof et al. (2018a), Yousof et al. (2018b), Brito et al. (2017), Hamedani et al. (2017), Cordeiro et al. (2018), Chakraborty et al. (2018), Hamedani et al. (2018), Korkmaz et al. (2018), Korkmaz et al. (2019) and Hamedani et al. (2019).

The rest of the paper is outlined as follows. In Section 2, we derive some mathematical properties for the new model including some stochastic properties, ordinary and incomplete moments, moments generating functions, residual life and reversed residual life functions, order statistics, quantile spread ordering, Rényi, Shannon and q-entropies. A simple type Copula based construction is derived in Section 3. Maximum likelihood estimation of the model parameters is addressed in Section 4. In Section 5, we assess the performance of the maximum likelihood estimators using a Monte Carlo simulation study. In Section 6, we provide two applications to real data to illustrate the importance of the GOGEIR model. Finally, some concluding remarks are presented in Section 7.

\section{Mathematical properties}

\subsection{Moments, cumulants and numerical results}

The $r^{\text {th }}$ ordinary moment of $W$ is given by

Then we obtain

$$
\mu_{r}^{\prime}=E\left(W^{r}\right)=\int_{-\infty}^{\infty} \omega^{r} f(\omega) d \omega
$$

$$
\left.\mu_{r}^{\prime}\right|_{(2>r)}=\sum_{j_{1}, j_{2}=0}^{\infty} v_{j_{1}, j_{2}}\left[\alpha\left(1+j_{1}+j_{2}\right)\right]^{\frac{r}{2}} K_{\left(1-\frac{r}{2}\right)}^{(\lambda, r)},
$$

where

$$
\begin{aligned}
& \left.\Gamma(1+\vartheta)\right|_{\left(\vartheta \in R^{+}\right)}=\vartheta ! \\
& =\prod_{h=0}^{\vartheta-1}(\vartheta-h) \\
& =\int_{0}^{\infty} t^{\vartheta} \exp (-t) d t,
\end{aligned}
$$

is the complete gamma function and

$$
K_{\Gamma(\cdot)}^{(\lambda, r)}=\lambda^{r} \Gamma(\cdot)
$$


. Setting $r=1$ in (9), we have the mean of $W$

$$
\mu_{1}^{\prime}=E(W)=K_{\left(1-\frac{1}{2}\right)}^{(\lambda, 1)} \sum_{j_{1}, j_{2}=0}^{\infty} v_{j_{1}, j_{2}} \sqrt{\alpha\left(1+j_{1}+j_{2}\right)}
$$

The last integration can be computed numerically for most parent distributions. The skewness and kurtosis measures can be calculated from the ordinary moments using wellknown relationships. The $n^{\text {th }}$ central moment of $W$, say $\mu_{n}$, follows as

$$
\mu_{n}=E(W-\mu)^{n}=\sum_{h=0}^{n}(-1)^{h}\left(\begin{array}{l}
n \\
h
\end{array}\right)\left(\mu_{1}^{\prime}\right)^{n} \mu_{n-h}^{\prime} .
$$

The cumulants $\left(\kappa_{n}\right)$ of $W$ follow recursively from

where

$$
\kappa_{n}=\mu_{n}^{\prime}-\sum_{r=0}^{n-1} \kappa_{r}\left(\begin{array}{c}
n-1 \\
r-1
\end{array}\right) \mu_{n-r}^{\prime}
$$

$$
\begin{aligned}
& \kappa_{1}=\mu_{1}^{\prime}, \\
& \kappa_{2}=\mu_{2}^{\prime}-\mu_{1}^{\prime 2}, \text { and } \\
& \kappa_{3}=\mu_{3}^{\prime}-3 \mu_{2}^{\prime} \mu_{1}^{\prime}+\mu_{1}^{\prime 3}
\end{aligned}
$$

The mean of $W$, variance $(\mathrm{V}(W))$ skewness $(\mathrm{S}(W))$ and kurtosis $(\mathrm{K}(W))$ measures also can be calculated from the ordinary moments using well-known relationships. The mean, variance, skewness and kurtosis of the GOGEIR distribution are computed numerically for some selected values using the $\mathrm{R}$ software. The numerical values displayed in Table 1 indicate that the skewness of the GOGEIR distribution is always positive and can range in the interval $(0.59,1.016)$. The spread for its kurtosis is ranging from 1.05 to 3.6. With fixing the values of $\beta$ and $\lambda$, we note that the $(\mathrm{S}(W))$ and $(\mathrm{K}(W))$ has no changes.

\begin{tabular}{|l|l|l|l|l|l|l|}
\hline \multicolumn{5}{|c|}{ Table 1: $\mathrm{E}(\mathrm{W}), \mathrm{V}(\mathrm{W}), \mathrm{S}(\mathrm{W})$ and $\mathrm{K}(\mathrm{W})$ of the GOGEIR distribution. } \\
\hline$\alpha$ & $\beta$ & $\lambda$ & $\mathrm{E}(\mathrm{W})$ & $\mathrm{V}(\mathrm{W})$ & $\mathrm{S}(\mathrm{W})$ & $\mathrm{K}(\mathrm{W})$ \\
\hline 1 & 3 & 5 & 7.331273 & 3.424783 & 0.6140166 & 3.488065 \\
\hline 2 & & & 10.36799 & 6.849566 & 0.6140166 & 3.488065 \\
\hline 5 & & & 16.39322 & 17.12391 & 0.6140166 & 3.488065 \\
\hline 10 & & & 23.18352 & 34.24783 & 0.6140166 & 3.488065 \\
\hline 50 & & & 51.83993 & 171.2391 & 0.6140166 & 3.488065 \\
\hline 100 & & & 73.31273 & 342.4783 & 0.6140166 & 3.488065 \\
\hline 500 & & & 163.9322 & 1712.391 & 0.6140166 & 3.488065 \\
\hline 1 & 10 & 6 & 10.89284 & 3.792974 & 0.6172596 & 3.63095 \\
\hline 10 & & & 34.44618 & 37.92974 & 0.6172596 & 3.63095 \\
\hline 50 & & & 77.02399 & 189.6487 & 0.6172598 & 3.63095 \\
\hline 100 & & & 108.9284 & 379.2974 & 0.6172596 & 3.63095 \\
\hline 100 & 1 & 10 & 111.757 & 1598.442 & 0.7912121 & 3.622083 \\
\hline & 10 & & 181.5473 & 1053.604 & 0.6172596 & 3.63095 \\
\hline & 50 & & 221.4869 & 758.3086 & 0.7064480 & 3.876657 \\
\hline & 100 & & 236.7447 & 672.022 & 0.7437847 & 3.974214 \\
\hline & 150 & & $4.0873 \times \mathrm{e}^{-6}$ & 60769.17 & 1.0162270 & 1.044547 \\
\hline 2 & 5 & 1 & 2.290886 & 0.2459378 & 0.599869 & 3.535318 \\
\hline & & 10 & 22.90886 & 24.59378 & 0.599869 & 3.535318 \\
\hline & & 100 & 229.0886 & 2459.378 & 0.599869 & 3.535318 \\
\hline
\end{tabular}




\begin{tabular}{|l|l|l|l|l|l|l|}
\hline & & 250 & 572.7216 & 15371.11 & 0.599869 & 3.535318 \\
\hline & & 500 & 1145.443 & 61484.45 & 0.599869 & 3.535318 \\
\hline
\end{tabular}

\subsection{Incomplete moment}

The $\mathrm{r}$ th incomplete moment, say $\phi_{r}(t)$, of $W$ can be expressed, from (8), as

$$
\begin{aligned}
& \phi_{r}(t)=\int_{-\infty}^{t} \omega^{r} f(\omega) d \omega \\
& =\left.\sum_{j_{1}, j_{2}=0}^{\infty} v_{j_{1}, j_{2}}\left[\alpha\left(1+j_{1}+j_{2}\right)\right]^{\frac{r}{2}} K_{\gamma\left(1-\frac{r}{2^{\prime}}\left[\alpha\left(1+j_{1}+j_{2}\right)\right]\left(\frac{\lambda}{t}\right)^{2}\right)}^{(\lambda, r)}\right|_{(2>r)}
\end{aligned}
$$

where $\gamma(\vartheta, u)$ is the incomplete gamma function.

$$
\begin{gathered}
\left.\gamma(\vartheta, u)\right|_{(\vartheta \neq 0,-1,-2, \ldots)}=\int_{0}^{u} t^{\vartheta-1} \exp (-t) d t \\
=\frac{u^{\vartheta}}{\vartheta}\left\{1 F_{1}[\vartheta ; \vartheta+1 ;-u]\right\} \\
=\sum_{\eta=0}^{\infty} \frac{(-1)^{\eta}}{\eta !(\vartheta+\eta)} u^{\vartheta+\eta}, \\
K_{\gamma(\cdot,)}^{(\lambda, r)}=\lambda^{r} \gamma(\cdot,)
\end{gathered}
$$

and $1 F_{1}[\because, \cdot]$ is a confluent hypergeometric function. The first incomplete moment can be calculated by stting $r=1$ in $\phi_{r}(t)$ as

$$
\phi_{1}(t)==\sum_{j_{1}, j_{2}=0}^{\infty} v_{j_{1}, j_{2}}\left[\alpha\left(1+j_{1}+j_{2}\right)\right]^{\frac{1}{2}} K_{\gamma\left(1-\frac{1}{2},\left[\alpha\left(1+j_{1}+j_{2}\right)\right]\left(\frac{\lambda}{t}\right)^{2}\right)}^{(\lambda, 1)} .
$$

\subsection{The moment generating function (MGF)}

The MGF $M_{W}(t)=E\left(e^{t W}\right)$ of $\omega$ can be derived from equation (4) as

$$
\left.M_{W}(t)\right|_{(2>r)}=\sum_{j_{1}, j_{2}, r=0}^{\infty} \frac{t^{r}}{r !} v_{j_{1}, j_{2}}\left[\alpha\left(1+j_{1}+j_{2}\right)\right]^{\frac{r}{2}} K_{\left(1-\frac{r}{2}\right)}^{(\lambda, r)} .
$$

Another alternative method for deriving the MGF can be introduced by the Wright generalized hypergeometric function (WHGF) which is defined by

$$
(\tau) \Psi_{(u)}\left[\begin{array}{l}
a_{1}, A_{1}, \ldots, a_{\tau}, A_{\tau} \\
b_{1}, B_{1}, \ldots, b_{u}, B_{u}
\end{array} ; \omega=\sum_{n=0}^{\infty} \frac{\prod_{j_{1}=1}^{\tau} \quad \Gamma\left(a_{j_{1}}+A_{j_{1}} n\right)}{\prod_{j_{1}=1}^{u} \Gamma\left(b_{j_{1}}+B_{j_{1}} n\right)} \frac{\omega^{n}}{n !}\right.
$$

Then, the MGF of (1) can be defined as

$$
M_{W}(t)={ }_{(1)} \Psi_{(0)}\left[\left(1,-\frac{1}{2}\right) ; \lambda t\right]
$$

Combining expressions (8) and (10), we obtain the MGF of the GOGEIR as

$$
M_{W}(t)=\sum_{j_{1}, j_{2}=0}^{\infty} v_{j_{1}, j_{2}}\left\{(1) \Psi_{(0)}\left[\left(1,-\frac{1}{2}\right) ; t \lambda \sqrt{\alpha\left(1+j_{1}+j_{2}\right)}\right]\right\} .
$$


Equations (9) and (11) can be easily evaluated by scripts of the Maple, Matlab and Mathematica platforms.

\subsection{Entropies}

The Rényi entropy of a random variable $W$ represents a measure of variation of the uncertainty. The Rényi entropy is defined by

$$
\left.R_{\vartheta}(W)\right|_{(\vartheta>0 \text { and } \vartheta \neq 1)}=\frac{\log \int_{-\infty}^{\infty} f(\omega)^{\vartheta} d \omega}{1-\vartheta}
$$

Using the PDF (6), we can write

$$
f(\omega)^{\vartheta}=\sum_{j_{1}, j_{2}=0}^{\infty} \eta_{j_{1}, j_{2}}^{(\vartheta)} \omega^{-3 \vartheta} \exp \left[-\left[\alpha\left(j_{1}+j_{2}+\vartheta\right)\right]\left(\frac{\lambda}{\omega}\right)^{2}\right],
$$

where

$$
\eta_{j_{1}, j_{2}}^{(\vartheta)}=\left(2 \alpha \beta \lambda^{2}\right)^{\vartheta} \frac{(-1)^{j_{1}+j_{2}}}{j_{1} !}\left(\begin{array}{c}
-\left(j_{1}+2\right) \\
j_{2}
\end{array}\right) \sum_{i=0}^{\infty}(-1)^{i}(i+\vartheta)^{j_{1}}\left(\begin{array}{c}
\vartheta(\beta-1) \\
i
\end{array}\right) .
$$

Then, the Rényi entropy of the GOGEIR model is given by

where

$$
\left.R_{\vartheta}(W)\right|_{(\vartheta>0 \text { and } \vartheta \neq 1)}=\frac{\log \left\{\sum_{j_{1}, j_{2}=0}^{\infty} \eta_{j_{1}, j_{2}}^{(\vartheta)}\left[(\vartheta) I_{0}^{\infty}\right]\right\}}{1-\vartheta}
$$

$$
(\vartheta) I_{0}^{\infty}=\int_{0}^{\infty} \omega^{-3 \vartheta} \exp \left\{-\left[\alpha\left(j_{1}+j_{2}+\vartheta\right)\right] \lambda^{2} \omega^{-2}\right\} d \omega
$$

The q-entropy, say $Q_{\delta}(W)$, can be obtained as

where

$$
\left.Q_{\delta}(W)\right|_{(\delta>0 \text { and } \delta \neq 1)}=\frac{\log \left(1-\left\{\sum_{j_{1}, j_{2}=0}^{\infty} \eta_{j_{1}, j_{2}}^{(\delta)}\left[(\delta) I_{0}^{\infty}\right]\right\}\right)}{\delta-1},
$$

$$
\eta_{j_{1}, j_{2}}^{(\delta)}=\left(2 \alpha \beta \lambda^{2}\right)^{\delta} \frac{(-1)^{j_{1}+j_{2}}}{j_{1} !}\left(\begin{array}{c}
-\left(j_{1}+2\right) \\
j_{2}
\end{array}\right) \sum_{i=0}^{\infty}(-1)^{i}(i+\delta)^{j_{1}}\left(\begin{array}{c}
\delta(\beta-1) \\
i
\end{array}\right),
$$

and

$$
(\delta) I_{0}^{\infty}=\int_{0}^{\infty} \omega^{-3 \delta} \exp \left\{-\left[\alpha\left(j_{1}+j_{2}+\delta\right)\right] \lambda^{2} \omega^{-2}\right\} d \omega
$$

The Shannon entropy of a random variable $W$, say $S \boldsymbol{E}$, is defined by

$$
S E=E\{-[\log f(W)]\} .
$$

It is the special case of the Rényi entropy, $\left.R_{\vartheta}(W)\right|_{(\vartheta>0}$ and $\left.\vartheta \neq 1\right)$, when $\vartheta \uparrow 1$.

\subsection{Order statistics}

Let $W_{1}, W_{2}, \ldots, W_{n}$ be a random sample (RS) from the GOGEIR distribution and let $W_{1}:{ }_{n}, W_{2}: n, \ldots, W_{n}: n$ be the corresponding order statistics. The PDF of the $i^{\text {th }}$ order statistic, say $W_{i}: n$, can be written as 


$$
f_{i}: n(\omega)=B^{-1}(i, 1+n-i) \sum_{j_{1}=0}^{n-i}(-1)^{j_{1}}\left(\begin{array}{c}
n-i \\
j_{1}
\end{array}\right) f(\omega) F^{j_{1}+i-1}(\omega),
$$

where B $(\cdot, \cdot)$ is the beta function. Substituting (1) and (2) in equation (12) and using a power series expansion, we have

where

$$
f(\omega) F(\omega)^{j_{1}+i-1}=\sum_{w, j_{2}=0}^{\infty} \tau_{w, j_{2}} h_{\alpha\left(w+j_{2}+1\right)}(\omega)
$$

$$
\tau_{w, j_{2}}=\frac{\alpha \beta(-1)^{w+j_{2}}}{w ![\alpha(w+m+1)]}\left(\begin{array}{c}
-(w+2) \\
j_{2}
\end{array}\right) \sum_{l=0}^{\infty}(-1)^{l}(l+1)^{w}\left(\begin{array}{c}
\beta\left(i+j_{1}\right)-1 \\
l
\end{array}\right) .
$$

Then, the PDF of $\omega_{i}: n$ can be expressed as

$$
f_{i}:{ }_{n}(\omega)=\sum_{j_{1}=0}^{n-i} \sum_{w, j_{2}=0}^{\infty}(-1)^{j_{1}} B^{-1}(i, 1+n-i)\left(\begin{array}{c}
n-i \\
j_{1}
\end{array}\right) \tau_{w, j_{2}} h_{\alpha\left(w+j_{2}+1\right)}(\omega) .
$$

The density function of the GOGEIR order statistics is a mixture of IR densities. Based on the last equation, the moments of $W_{i}: n$ can be expressed as

$$
\left.E\left(W_{i}^{\delta}: n\right)\right|_{(2>\delta)}=\sum_{w, j_{2}=0}^{\infty} \sum_{j_{1}=0}^{n-i} \frac{(-1)^{j_{1}} B^{-1}(i, 1+n-i)}{\left[\alpha\left(w+j_{2}+1\right)\right]^{\frac{-\delta}{2}}}\left(\begin{array}{c}
n-i \\
j_{1}
\end{array}\right) \tau_{w, j_{2}} K_{\left(1-\frac{\delta}{2}\right)}^{(\lambda, \delta)} .
$$

\subsection{Quantile spread order}

The quantile spread $(Y .(\vartheta))$ of the rv $W \sim \operatorname{GOGEIR}(\alpha, \beta, \lambda)$ having the $\operatorname{CDF}(5)$ is given by

which implies

$$
\left.\Upsilon_{\omega}(\vartheta)\right|_{\left(\vartheta \in\left(\frac{1}{2}, 1\right)\right)}=\left[F^{-1}(\vartheta)\right]-\left[F^{-1}(1-\vartheta)\right]
$$

$$
\Upsilon_{\omega}(\vartheta)=\left[S^{-1}(1-\vartheta)\right]-\left[S^{-1}(\vartheta)\right],
$$

where $F^{-1}(\boldsymbol{\vartheta})=S^{-1}(1-\boldsymbol{\vartheta})$ and $S(\cdot)=1-F(\cdot)$ is the survival function. The $Y$. $\vartheta)$ of a distribution describes how the probability mass is placed symmetrically about its median and hence can be used to formalize concepts such as peakedness and tail weight traditionally associated with kurtosis. So, it allows us to separate concepts of kurtosis and peakedness for asymmetric models.

Let $Z_{1}$ and $\omega_{2}$ be two random variables following the GOGEIR model with quantile spreads $Y_{Z_{1}}$ and $Y_{Z_{2}}$, respectively. Then $Z_{1}$ is called smaller than $Z_{2}$ in quantile spread order, denoted as

when

$$
Z_{1} \leq_{[\gamma]} Z_{2}
$$

$$
Y_{Z_{1}}(\vartheta) \leq\left. Y_{Z_{2}}(\vartheta)\right|_{\left(\vartheta \in\left(\frac{1}{2}, 1\right)\right)}
$$

The following properties of the quantile spread order can be obtained:

- The order $\leq_{[r]}$ is location-Free 


$$
Z_{1} \leq_{[\gamma]} Z_{2} \text { if }\left(Z_{1}+\eta\right) \leq_{[\gamma]} Z_{2} \forall \eta \in R
$$

- The order $\leq_{[Y]}$ is dilative

$$
Z_{1} \leq_{[\gamma]} \eta Z_{1} \text { whenever } \eta \geq 1 \text { and } Z_{2} \leq\left._{[\gamma]} \eta Z_{2}\right|_{\eta \geq 1}
$$

- Let $F_{Z_{1}}$ and $F_{Z_{2}}$ be symmetric, then

$$
Z_{1} \leq_{[\gamma]} Z_{2} \text { if, and only if } F_{Z_{1}}^{-1}(\vartheta) \leq F_{Z_{2}}^{-1}(\vartheta), \forall \vartheta \in\left(\frac{1}{2}, 1\right) \text {. }
$$

- The order $\leq_{[r]}$ implies ordering of the mean absolute deviation around the median, say $\omega(\cdot)$,

and

where

$$
\omega\left(Z_{1}\right)=E\left[\left|Z_{1}-\operatorname{Median}\left(Z_{1}\right)\right|\right]
$$

Finally

$$
\omega\left(Z_{2}\right)=E\left[\left|Z_{2}-\operatorname{Median}\left(Z_{2}\right)\right|\right],
$$

$$
Z_{1} \leq_{[\gamma]} Z_{2} \Rightarrow \omega\left(Z_{1}\right) \leq_{[\gamma]} \omega\left(Z_{2}\right)
$$

$$
Z_{1} \leq_{[Y]} Z_{2} \text { if, and only if }-Z_{1} \leq_{[\gamma]}-Z_{2} \text {. }
$$

\subsection{Residual life and reversed residual life functions}

The $n^{\text {th }}$ moment of the residual life, say

$$
a_{n}(t)=E\left[(W-t)^{n}\right] \mid(W>t \text { and } n=1,2, \ldots),
$$

uniquely determines the CDF $F(\omega)$. The $n^{\text {th }}$ moment of the residual life of $W$ is given by

Therefore

$$
a_{n}(t)=\frac{\int_{t}^{\infty}(\omega-t)^{n} d F(\omega)}{1-F(t)}
$$

$$
\left.a_{n}(t)\right|_{(2>n)}=\frac{1}{1-F(t)} \sum_{j_{1}, j_{2}=0}^{\infty} \varphi_{j_{1}, j_{2}}\left[\alpha\left(1+j_{1}+j_{2}\right)\right]_{\Gamma\left(1-\frac{n}{2},\left[\alpha\left(1+j_{1}+j_{2}\right)\right]\left(\frac{n}{t}\right)^{\frac{n}{2}} K^{2}\right)^{(\lambda, n)}}
$$

where

$$
\begin{gathered}
\varphi_{j_{1}, j_{2}}=v_{j_{1}, j_{2}} \sum_{r=0}^{n}(-t)^{n-r}\left(\begin{array}{l}
n \\
r
\end{array}\right), \\
\left.\Gamma(\vartheta, u)\right|_{(\omega>0)}=\int_{u}^{\infty} t^{\vartheta-1} \exp (-t) d t \\
\Gamma(\vartheta, u)=\Gamma(\omega)-\gamma(\vartheta, u)
\end{gathered}
$$

and

$$
K_{\Gamma(\cdot,)}^{(\lambda, n)}=\lambda^{n} \Gamma(\cdot, \cdot)
$$

Another interesting function is the mean residual life (MRL) function or the life expectation at age $t$ defined by 


$$
z_{1}(t)=E\left[(W-t)^{n}\right] \mid(W>t \text { and } n=1),
$$

which represents the expected additional life length for a unit which is alive at age $t$. The MRL of $W$ can be obtained by setting $n=1$ in the last equation. The $n^{\text {th }}$ moment of the reversed residual life, say

$$
\left.Z_{n}(t)=\left.E\left[(t-W)^{n}\right]\right|_{(W \leq t, t>0} \text { and } n=1,2, \ldots\right)
$$

uniquely determines the CDF $F(\omega)$. We obtain

$$
A_{n}(t)=\frac{\int_{0}^{t}(t-\omega)^{n} d F(\omega)}{F(t)} .
$$

Then, the $n^{\text {th }}$ moment of the reversed residual life of $W$ becomes

where

$$
\left.A_{n}(t)\right|_{(2>n)}=\frac{1}{F(t)} \sum_{j_{1}, j_{2}=0}^{\infty} \phi_{j_{1}, j_{2}}\left[\alpha\left(1+j_{1}+j_{2}\right)\right]_{\gamma\left(1-\frac{n}{2},\left[\alpha\left(1+j_{1}+j_{2}\right)\right]\left(\frac{\lambda}{t}\right)^{2}\right)^{\prime}}^{(\lambda, n)}
$$

$$
\phi_{j_{1}, j_{2}}=v_{j_{1}, j_{2}} \sum_{r=0}^{n}(-1)^{r} t^{n-r}\left(\begin{array}{l}
n \\
r
\end{array}\right) .
$$

The mean inactivity time (MIT) or mean waiting time (MWT) also called the mean reversed residual life function is given by

$$
A_{1}(t)=E\left[(t-W)^{n}\right] \mathrm{I}_{(W \leq t, t>0 \text { and } n=1)}
$$

and it represents the waiting time elapsed since the failure of an item on condition that occurred in $(0, t)$. The MIT of the GOGEIR distribution can be obtained easily by setting $n=1$

in

the

above

equation.

\section{Simple type Copula based construction}

In this Section, we consider several approaches to construct the bivariate and the multivariate GOGEIR type distributions via copula (or with straightforward bivariate CDFs form, in which we just need to consider two different GOGEIR CDFs).

\subsection{Via Morgenstern family}

First, we start with CDF for Morgenstern family of two random variables $\left(W_{1}, W_{2}\right)$ which has the following form

setting

$$
\left.F_{\lambda}\left(W_{1}, \omega_{2}\right)\right|_{(|\lambda| \leq 1)}=F_{1}\left(\omega_{1}\right) F_{2}\left(\omega_{2}\right)\left\{1+\lambda\left[1-F_{1}\left(\omega_{1}\right)\right]\left[1-F_{2}\left(\omega_{2}\right)\right]\right\},
$$

and

$$
F_{\alpha_{1}, \beta_{1}, \lambda}\left(\omega_{1}\right)=\left\{1-\exp \left[\frac{-\exp \left(-\alpha_{1} \lambda^{2} \omega_{1}^{-2}\right)}{1-\exp \left(-\alpha_{1} \lambda^{2} \omega_{1}^{-2}\right)}\right]\right\}^{\beta_{1}},
$$

$$
F_{\alpha_{2}, \beta_{2}, \lambda}\left(\omega_{2}\right)=\left\{1-\exp \left[\frac{-\exp \left(-\alpha_{2} \lambda^{2} \omega_{2}^{-2}\right)}{1-\exp \left(-\alpha_{2} \lambda^{2} \omega_{2}^{-2}\right)}\right]\right\}^{\beta_{2}},
$$

then we have a seven-dimension parameter model. The estimation will be a big issue here. Estimation via Bayesian paradigm may be done, but again, the choice of appropriate priors will be challenging. 


\subsection{Via Clayton copula}

\section{The bivariate extension}

The bivariate extension via Clayton copula can be considered as a weighted version of the Clayton copula, which is of the form

$$
C(u, v)=\left[u^{-\left(\delta_{1}+\delta_{2}\right)}+v^{-\left(\delta_{1}+\delta_{2}\right)}-1\right]^{-\frac{1}{\delta_{1}+\delta_{2}}}
$$

This is indeed a valid copula. Next, let us assume that $W \sim \operatorname{GOGEIR}\left(\alpha_{1}, \beta_{1}, \lambda\right)$ and $Y \sim$ $\operatorname{GOGEIR}\left(\alpha_{2}, \beta_{2}, \lambda\right)$. Then, setting

and

$$
u=\left\{1-\exp \left[\frac{-\exp \left(-\alpha_{1} \lambda^{2} \omega_{1}^{-2}\right)}{1-\exp \left(-\alpha_{1} \lambda^{2} \omega_{1}^{-2}\right)}\right]\right\}^{\beta_{1}}
$$

$$
v=F(y)=\left\{1-\exp \left[\frac{-\exp \left(-\alpha_{2} \lambda^{2} y_{2}^{-2}\right)}{1-\exp \left(-\alpha_{2} \lambda^{2} y_{2}^{-2}\right)}\right]\right\}^{\beta_{2}}
$$

the associated CDF bivariate GOGEIR type distribution will be

$$
H(\omega, y)=\left(\begin{array}{c}
\left.\left\{1-\exp \left[\frac{-\exp \left(-\alpha_{1} \lambda^{2} \omega_{1}^{-2}\right)}{1-\exp \left(-\alpha_{1} \lambda^{2} \omega_{1}^{-2}\right)}\right]\right\}^{-\beta_{1}\left(\delta_{1}+\delta_{2}\right)}\right)^{-\frac{1}{\delta_{1}+\delta_{2}}} \\
+\left\{1-\exp \left[\frac{-\exp \left(-\alpha_{2} \lambda^{2} y_{1}^{-2}\right)}{1-\exp \left(-\alpha_{2} \lambda^{2} y_{2}^{-2}\right)}\right]\right\}^{-\beta_{2}\left(\delta_{1}+\delta_{2}\right)}
\end{array}\right)^{-1} .
$$

Note: Depending on the specific baseline CDF, one may construct various bivariate GOGEIR type model in which $\left(\delta_{1}+\delta_{2}\right) \geq 0$.

\section{The Multivariate extension}

A straightforward $d$-dimensional extension from the above will be

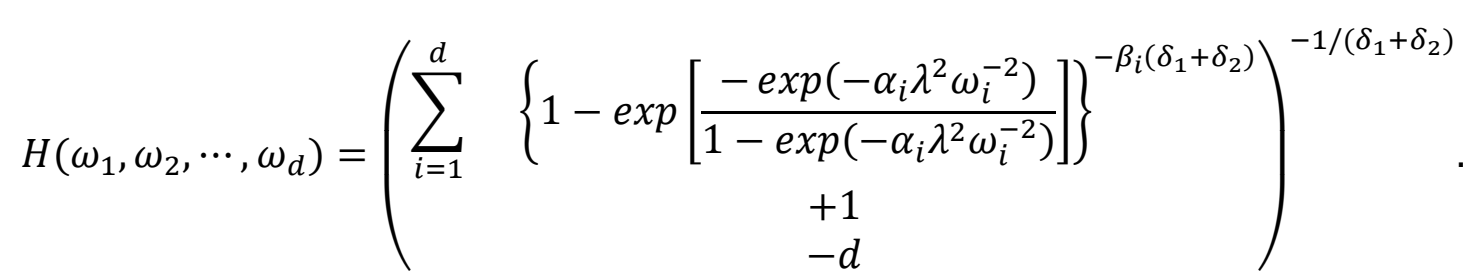

Further future works could be be allocated for studying the bivariate and the multivariate extensions of the GOGEIR model.

\subsection{Some stochastic properties}

Suppose $W_{1} \sim \operatorname{GOGEIR}\left(\alpha_{1}, \beta_{1}, \lambda\right)$ and $W_{2} \sim \operatorname{GOGEIR}\left(\alpha_{2}, \beta_{2}, \lambda\right)$. Then $W_{1}$ is stochastically smaller than $W_{2}$ if $\alpha_{1}>\alpha_{2}$ and $\beta_{1}>\beta_{2}$. Note that for any $\alpha_{1}>\alpha_{2}$,

$$
\exp \left(-\alpha_{1} \lambda^{2} \omega_{1}^{-2}\right)>\exp \left(-\alpha_{2} \lambda^{2} \omega_{2}^{-2}\right) \text {. }
$$

This is true for both integer and fractional values of $\alpha_{1}$ and $\alpha_{2}$. After some algebra, we get the following: Since for $\alpha_{1}>\alpha_{2}$ we have

then

$$
\exp \left(-\alpha_{1} \lambda^{2} \omega_{1}^{-2}\right)>\exp \left(-\alpha_{2} \lambda^{2} \omega_{2}^{-2}\right)
$$

$$
\left\{1-\exp \left(-\alpha_{1} \lambda^{2} \omega_{1}^{-2}\right)\right\}<\left\{1-\exp \left(-\alpha_{2} \lambda^{2} \omega_{2}^{-2}\right)\right\}
$$


and

$$
\frac{\exp \left(-\alpha_{1} \lambda^{2} \omega_{1}^{-2}\right)}{1-\exp \left(-\alpha_{1} \lambda^{2} \omega_{1}^{-2}\right)}>\frac{\exp \left(-\alpha_{2} \lambda^{2} \omega_{2}^{-2}\right)}{1-\exp \left(-\alpha_{2} \lambda^{2} \omega_{2}^{-2}\right)}
$$

$$
\frac{-\exp \left(-\alpha_{1} \lambda^{2} \omega_{1}^{-2}\right)}{1-\exp \left(-\alpha_{1} \lambda^{2} \omega_{1}^{-2}\right)}<\frac{-\exp \left(-\alpha_{2} \lambda^{2} \omega_{2}^{-2}\right)}{1-\exp \left(-\alpha_{2} \lambda^{2} \omega_{2}^{-2}\right)}
$$

Rest of the proof follows immediately from here

$$
\begin{aligned}
& -\frac{\exp \left(-\alpha_{1} \lambda^{2} \omega_{1}^{-2}\right)}{1-\exp \left(-\alpha_{1} \lambda^{2} \omega_{1}^{-2}\right)}<-\frac{\exp \left(-\alpha_{2} \lambda^{2} \omega_{2}^{-2}\right)}{1-\exp \left(-\alpha_{2} \lambda^{2} \omega_{2}^{-2}\right)} \\
& \rightarrow\left(1-\exp \left\{\frac{-\exp \left(-\alpha_{1} \lambda^{2} \omega_{1}^{-2}\right)}{1-\exp \left(-\alpha_{1} \lambda^{2} \omega_{1}^{-2}\right)}\right\}\right)^{\beta_{1}}>\left(1-\exp \left\{\frac{-\exp \left(-\alpha_{2} \lambda^{2} \omega_{2}^{-2}\right)}{1-\exp \left(-\alpha_{2} \lambda^{2} \omega_{2}^{-2}\right)}\right\}\right)^{\beta_{2}} \\
& \rightarrow 1-\left(1-\exp \left\{\frac{-\exp \left(-\alpha_{1} \lambda^{2} \omega_{1}^{-2}\right)}{1-\exp \left(-\alpha_{1} \lambda^{2} \omega_{1}^{-2}\right)}\right\}\right)^{\beta_{1}} \\
& <1-\left(1-\exp \left\{\frac{-\exp \left(-\alpha_{2} \lambda^{2} \omega_{2}^{-2}\right)}{1-\exp \left(-\alpha_{2} \lambda^{2} \omega_{2}^{-2}\right)}\right\}\right)^{\beta_{2}} .
\end{aligned}
$$

This completes the proof.

\section{Estimation}

Let $\omega_{1}, \ldots, \omega_{n}$ be a RS IRom the GOGEIR distribution with parameters $\alpha, \beta, \lambda$ and 2 . Let $\Phi=(\alpha, \beta, \lambda)$ ? be the $4 \times 1$ parameter vector. For determining the MLE of $\Theta$, we have the $\log$-likelihood function

$$
\begin{aligned}
& \ell(\Phi)_{n}=n \log \alpha+n \log \beta+n \log 2+2 n \log \lambda+ \\
& -3 \sum_{i=1}^{n} \log \omega_{i}-2 \sum_{i=1}^{n} \log \left[1-\eta_{i}^{(\alpha, \lambda)}\right] \\
& +\sum_{i=1}^{n}\left[\frac{-\eta_{i}^{(\alpha, \lambda)}}{1-\eta_{i}^{(\alpha, \lambda)}}\right]+\sum_{i=1}^{n} \log \left[\eta_{i}^{(\alpha, \lambda)}\right] \\
& +(\beta-1) \sum_{i=1}^{n} \log \left(1-\exp \left[\frac{-\eta_{i}^{(\alpha, \lambda)}}{1-\eta_{i}^{(\alpha, \lambda)}}\right]\right)
\end{aligned}
$$

where $\eta_{i}^{(\alpha, \lambda)}=\exp \left(-\alpha \lambda^{2} \omega_{i}^{-2}\right)$. The components of the score vector

$$
U(\Phi)=\frac{\partial \ell}{\partial \Phi}=\left(U_{\alpha}=\frac{\partial \ell(\Phi)}{\partial \alpha}, U_{\beta}=\frac{\partial \ell(\Phi)}{\partial \beta}, U_{\lambda}=\frac{\partial \ell(\Phi)}{\partial \lambda}\right)^{?},
$$

are easy to derive, setting the nonlinear system of equations $U_{\alpha}=U_{\beta}=U_{\lambda}=0$ and $U_{2}=$ 0 and solving them simultaneously yields the MLE To solve these equations, it is usually more convenient to use nonlinear optimization methods such as the quasi-Newton algorithm to numerically maximize $\ell(\Phi)$. Further works could be devoted using other different methods to estimate the GOGEIR parameters such as least squares, moments, weighted least squares, Anderson-Darling, Jackknife, bootstrap, Cramér-vonMises, Bayesian analysis, among others, and compare the estimators based on these methods. 


\section{Simulations}

Using (7), we simulate the GOGEIR model via taking $n=20,50,150,500$ and 1000 . We evaluate the MLEs of the parameters for each sample size. Then, repeating this process $N=1000$ times and calculate the averages of the estimates (AEs), mean squared errors (MSEs). Table 2 gives all simulation results. The numerical results in Table 2 indicate that MSEs of $\stackrel{k}{\varepsilon}, \epsilon$ and $\xi^{k}$ decay toward 0 when $n$ increases for all initial values of of $\alpha, \beta$ and $\lambda$. The AEs of the parameters tend to be closer to the true (initial) parameter values (I: $\alpha=1.75, \beta=1.5$ and $\lambda=2.25$ and II: $\alpha=2.5, \beta=2.25$ and $\lambda=1.25$ ) when $n$ increases. These results supports that the asymptotic normal model provides an adequate approximation to the finite sample distribution of the MLEs. Table 2 below gives the AEs and MSEs based on $N=1000$ simulations of the GOGEIR model for some values of $\alpha, \beta$ and $\lambda$.

\begin{tabular}{|l|l|l|l|l|l|l|l|l|l|l|}
\hline \multicolumn{7}{|c|}{ Table 2: The AEs and MSEs based on N=1000 simulations. } \\
\hline $\mathrm{n}$ & & $\Phi$ & AEs & & MSEs & & $\Phi$ & AEs & & MSEs \\
\hline & $\mathrm{I}$ & & & & & II & & & & \\
\hline 20 & & $\alpha$ & 1.96717 & & 0.91203 & & $\alpha$ & 2.45471 & & 1.05401 \\
\hline & & $\beta$ & 1.88852 & & 0.02123 & & $\beta$ & 2.45784 & & 0.19173 \\
\hline & & $\lambda$ & 2.47091 & & 0.04563 & & $\lambda$ & 1.22858 & & 0.13019 \\
\hline 50 & & $\alpha$ & 1.73284 & & 0.29813 & & $\alpha$ & 2.55139 & & 0.44856 \\
\hline & & $\beta$ & 1.81745 & & 0.00789 & & $\beta$ & 2.35185 & & 0.11015 \\
\hline & & $\lambda$ & 2.35153 & & 0.01607 & & $\lambda$ & 1.24736 & & 0.10311 \\
\hline 150 & & $\alpha$ & 1.74199 & & 0.18674 & & $\alpha$ & 2.52722 & & 0.11051 \\
\hline & & $\beta$ & 1.55308 & & 0.00283 & & $\beta$ & 2.26009 & & 0.01125 \\
\hline & & $\lambda$ & 2.29044 & & 0.00526 & & $\lambda$ & 1.248323 & & 0.03483 \\
\hline & & & & & & & & & & \\
\hline 500 & & $\alpha$ & 1.75981 & & 0.02537 & & $\alpha$ & 2.49993 & & 0.04112 \\
\hline & & $\beta$ & 1.50158 & & 0.00081 & & $\beta$ & 2.25033 & & 0.00312 \\
\hline & $\lambda$ & 2.25266 & & 0.00162 & & $\lambda$ & 1.24845 & & 0.01164 \\
\hline & & & & & & & & & & \\
\hline 1000 & & $\alpha$ & 1.75051 & & 0.00229 & & $\alpha$ & 2.500942 & & 0.00493 \\
\hline & & $\beta$ & 1.50012 & & 0.00045 & & $\beta$ & 2.250011 & & 0.00003 \\
\hline & $\lambda$ & 2.25039 & & 0.00083 & & $\lambda$ & 1.250023 & & 0.00081 \\
\hline
\end{tabular}

\section{Real data modeling}

This section presents two applications of the new distribution using real data sets. We shall compare the fit of the new distribution with the Weibull Inverse Weibull (W-IW), exponentiated IW (E-IW), Kumaraswamy IW(Kum-IW), beta IW (B-IW) transmuted IW (T-IW), gamma extended IW (GE-IW), Marshall-Olkin IW (MO-IW), MOKum-IW, generalized MO-IW(GMO-IW), KumMO-IW and IW distributions. The PDFs of the competitive model are available in statistical literature. The unknown parameters of the above PDFs are all positive real numbers except for the T-IW distribution for which $|\lambda| \leq$ 1 .

The 1 st data set consists of 100 observations of breaking stress of carbon fibers given by Nichols and Padgett (2006) \{0.920, 0.9280, 0.997, 0.9971, 1.0610, 1.117, 1.1620, 1.183, $1.187,1.1920,1.196,1.2130,1.215,1.2199,1.220,1.2240,1.225,1.2280,1.237,1.240$, 
$1.244,1.259,1.2610,1.263,1.276,1.310,1.3210,1.3290,1.3310,1.337,1.351,1.359$, $1.388,1.4080,1.449,1.4497,1.450,1.459,1.471,1.475,1.477,1.480,1.489,1.501,1.507$, $1.515,1.530,1.5304,1.533,1.544,1.5443,1.552,1.556,1.5620,1.566,1.585,1.586$, $1.599,1.602,1.6140,1.6160,1.617,1.6280,1.6840,1.7110,1.7180,1.733,1.7380$, $1.7430,1.7590,1.777,1.7940,1.799,1.806,1.814,1.8160,1.8280,1.830,1.884,1.892$, $1.944,1.972,1.9840,1.987,2.02,2.0304,2.0290,2.0350,2.0370,2.0430,2.0460,2.0590$, $2.111,2.165,2.686,2.778,2.972,3.504,3.863,5.3060\}$.

The 2 nd data set consists of 63 observations of the strengths of $1.5 \mathrm{~cm}$ glass fibers (see Smith and Naylor (1987)) $\{1.312,1.314,1.479,1.552,1.700,1.803,1.861,1.865,1.944$, $1.958,1.966,1.997,2.006,2.021,2.027,2.055,2.063,2.098,2.140,2.179,2.224,2.240$, $2.253,2.270,2.272,2.274,2.301,2.301,2.359,2.382,2.382,2.426,2.434,2.435,2.478$, $2.490,2.511,2.514,2.535,2.554,2.566,2.570,2.586,2.629,2.633,2.642,2.648,2.684$, 2.697, 2.726, 2.770, 2.773, 2.800, 2.809, 2.818, 2.821, 2.848, 2.880, 2.809, 2.818, 2.821, $2.848,2.880,2.954,3.012$, 3.067, 3.084, 3.090, 3.096, 3.128, 3.233, 3.433, 3.585, 3.585\}. In order to compare the distributions, we consider the following criteria: the (Maximized Log-Likelihood), AIc (Akaike Information Criterion), CAI $c$ (Consistent Akaike Information Criterion), Bc (Bayesian information criterion) and HQIc (HannanQuinn information Criterion). The model with minimum values for these statistics could be chosen as the best model to fit the data. Total time test (TTT) plot (see Aarset (1987) anf Figure 1) is an important graphical approach to verify whether our data can be applied to a specific model or not. The TTT plots the two real data sets is presented in Figure 1. This plot indicates that the empirical HRFs of the the two data sets are increasing.

Table 3: The statistics AIC, BIC, HQIC and CAIC values for breaking stress data.

\begin{tabular}{|l|l|l|l|l|}
\hline Model & \multicolumn{5}{|c|}{ Measures } \\
\hline & AIC & BIC & HQIC & CAIC \\
\hline GOGEIR & 143.76 & 151.58 & 146.92 & 144.01 \\
\hline W-IW & 294.5 & 304.9 & 298.7 & 294.9 \\
\hline E-IW & 295.7 & 303.5 & 298.9 & 296.0 \\
\hline Kum-IW & 297.1 & 307.5 & 301.3 & 297.5 \\
\hline B-IW & 311.1 & 321.6 & 315.4 & 311.6 \\
\hline GE-IW & 312.0 & 332.4 & 316.2 & 312.4 \\
\hline IW & 348.3 & 353.5 & 350.4 & 348.4 \\
\hline T-IW & 350.5 & 358.3 & 353.6 & 350.7 \\
\hline MO-IW & 351.3 & 359.1 & 354.5 & 351.6 \\
\hline
\end{tabular}

Table 4: MLEs and their standard errors (in parentheses) for breaking stress of carbon fiber data.

\begin{tabular}{|l|l|l|l|l|}
\hline Model & \multicolumn{4}{|c|}{ Estimates } \\
\hline GOGEIR $(\alpha, \beta, \lambda)$ & 0.88 & 1.903 & 1.36 & \\
\hline & $(0.000)$ & $(0.44)$ & $(0.000)$ & \\
\hline W-IW $(\alpha, \beta, \mathrm{a}, \mathrm{b})$ & 2.2231 & 0.355 & 6.9721 & 4.9179 \\
\hline & $(11.409)$ & $(0.411)$ & $(113.811)$ & $(3.756)$ \\
\hline Kum-IW $(\alpha, \beta, \mathrm{a}, \mathrm{b})$ & 2.0556 & 0.4654 & 6.2815 & 224.18 \\
\hline & $(0.071)$ & $(0.00701)$ & $(0.063)$ & $(0.164)$ \\
\hline B-IW $(\alpha, \beta, \mathrm{a}, \mathrm{b})$ & 1.6097 & 0.4046 & 22.0143 & 29.7617 \\
\hline
\end{tabular}


Wahhab Salim Mohammed, Rania H. M. Abdelkhalek \& S. I. Ansari

\begin{tabular}{|l|l|l|l|l|}
\hline & $(2.498)$ & $(0.108)$ & $(21.432)$ & $(17.479)$ \\
\hline GE-IW $(\alpha, \beta, \mathrm{a}, \mathrm{b})$ & 1.3692 & 0.4776 & 27.6452 & 17.4581 \\
\hline & $(2.017)$ & $(0.133)$ & $(14.136)$ & $(14.818)$ \\
\hline E-IW $(\alpha, \beta, \mathrm{a})$ & 69.1489 & 0.5019 & 145.3275 & \\
\hline T-IW $(\alpha, \beta, \lambda)$ & $(57.349)$ & $(0.08)$ & $(122.924)$ & \\
\hline & 1.9315 & 1.7435 & 0.0819 & \\
\hline MO-IW $(\alpha, \beta, \mathrm{a})$ & $(0.097)$ & $(0.076)$ & $(0.198)$ & \\
\hline & 2.3066 & 1.5796 & 0.5988 & \\
\hline IW $(\alpha, \beta)$ & $(0.498)$ & $(0.16)$ & $(0.3091)$ & \\
\hline & 1.8705 & 1.7766 & & \\
\hline & $(0.112)$ & $(0.113)$ & & \\
\hline
\end{tabular}

Table 5: The statistics AI_c, BI_c, HQI_c and CAI_c values for glass fiber data.

\begin{tabular}{|l|l|l|l|l|}
\hline Model & \multicolumn{5}{|c|}{ Measures } \\
\hline & AIC & BIC & HQIC & CAIC \\
\hline GOGEIR & 65.72 & 72.15 & 68.25 & 66.13 \\
\hline B-IW & 68.6 & 77.2 & 72.0 & 69.3 \\
\hline GE-IW & 69.6 & 78.1 & 72.9 & 70.3 \\
\hline IW & 97.7 & 102 & 99.4 & 97.9 \\
\hline T-IW & 100.1 & 106.5 & 102.6 & 100.5 \\
\hline MO-IW & 101.7 & 108.2 & 104.2 & 102.1 \\
\hline
\end{tabular}

Table 6: MLEs and their standard errors for glass fiber data.

\begin{tabular}{|l|l|l|l|l|}
\hline Model & \multicolumn{4}{|c|}{ Estimates } \\
\hline GOGEIR $(\alpha, \beta, \lambda)$ & 1.013 & 3.95 & 1.042 & \\
\hline & $(4.77)$ & $(1.23)$ & $(2.46)$ & \\
\hline B-IW $(\alpha, \beta, a, b)$ & 2.0518 & 0.647 & 15.076 & 36.94 \\
\hline GE-IW $(\alpha, \beta, \mathrm{a}, \mathrm{b})$ & $(0.986)$ & $(0.163)$ & $(12.06)$ & $(22.65)$ \\
\hline & 1.663 & 0.7421 & 32.112 & 13.269 \\
\hline T-IW $(\alpha, \beta, \mathrm{a})$ & $(0.95)$ & $(0.197)$ & $(17.397)$ & $(9.967)$ \\
\hline & 1.3068 & 2.79 & 0.13 & \\
\hline MO-IW $(\alpha, \beta, \mathrm{a})$ & $(0.034)$ & $(0.17)$ & $(0.21)$ & \\
\hline & 1.544 & 2.388 & 0.482 & \\
\hline IW $(\alpha, \beta)$ & $(0.226)$ & $(0.253)$ & $(0.25)$ & \\
\hline & 1.264 & 2.888 & & \\
\hline
\end{tabular}




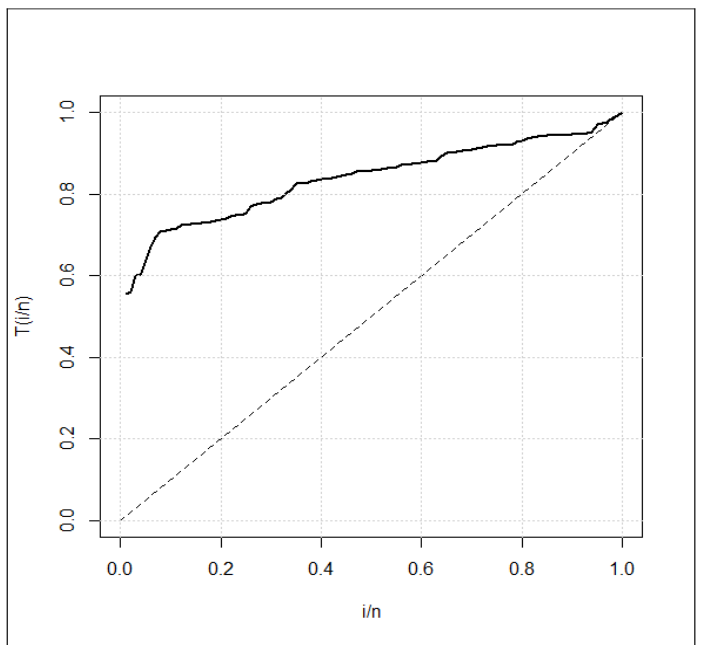

The $1^{\text {st }}$ data.

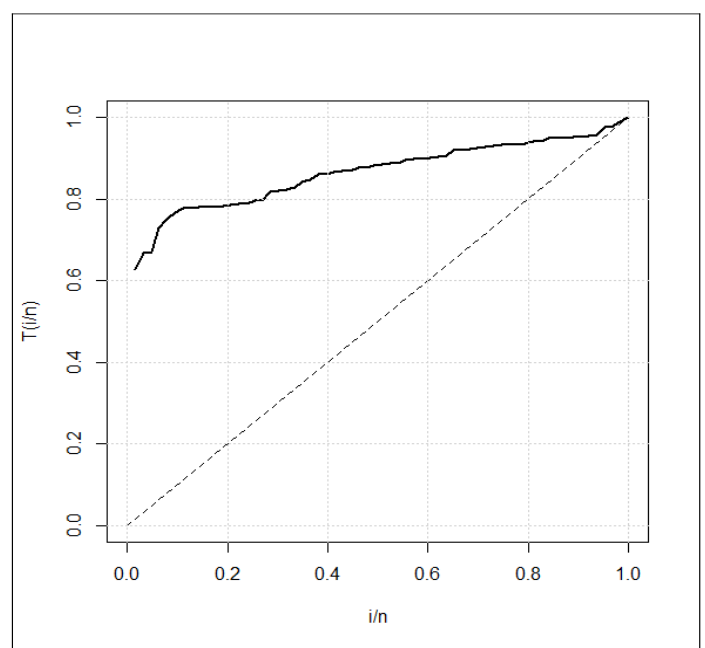

The $2^{\text {nd }}$ data.

Figure 1: TTT plots.

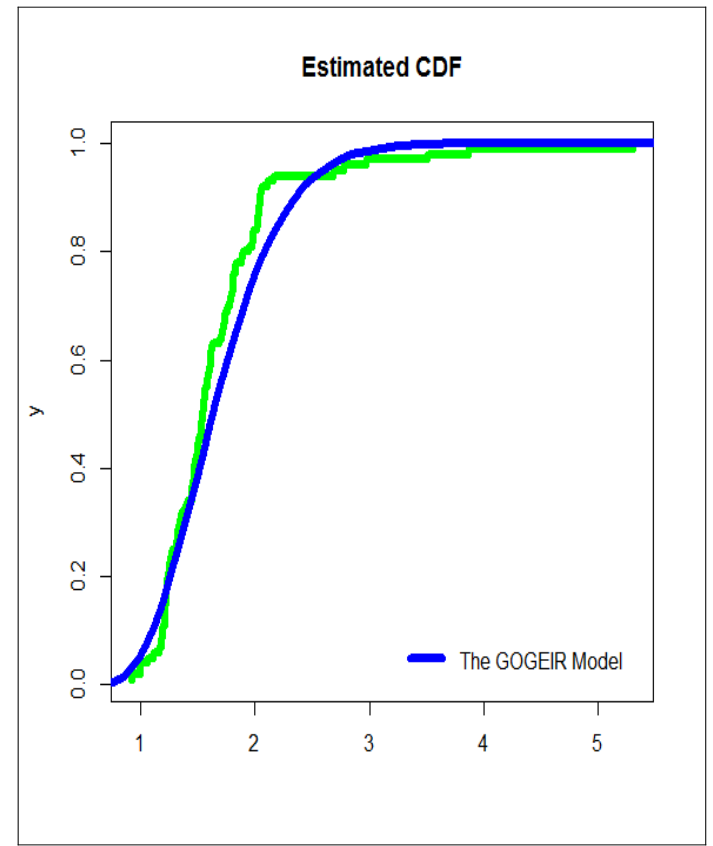

The $1^{\text {st }}$ data.

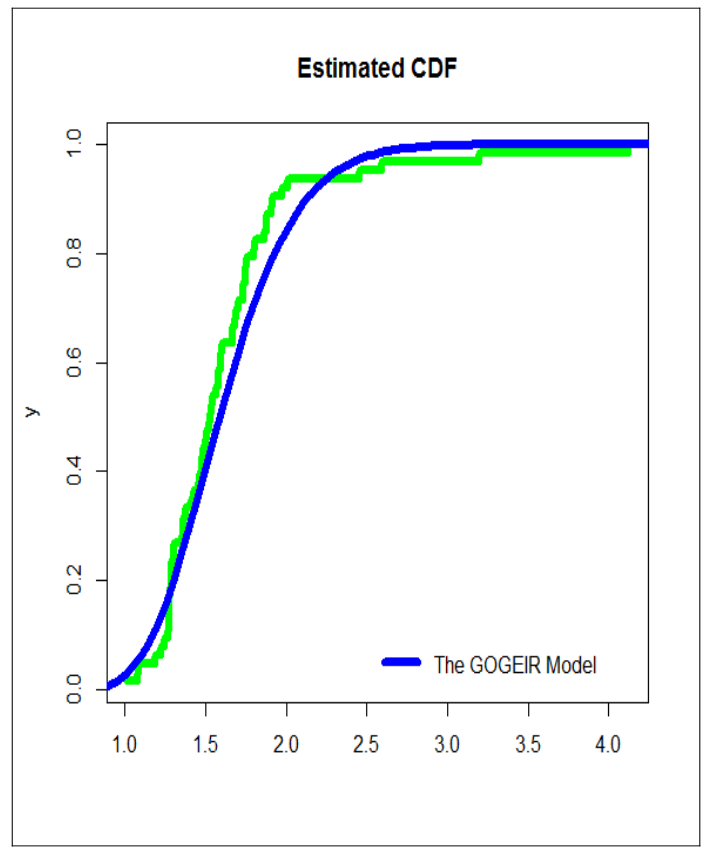

The $2^{\text {nd }}$ data.

Figure 2: Estimated CDFs. 


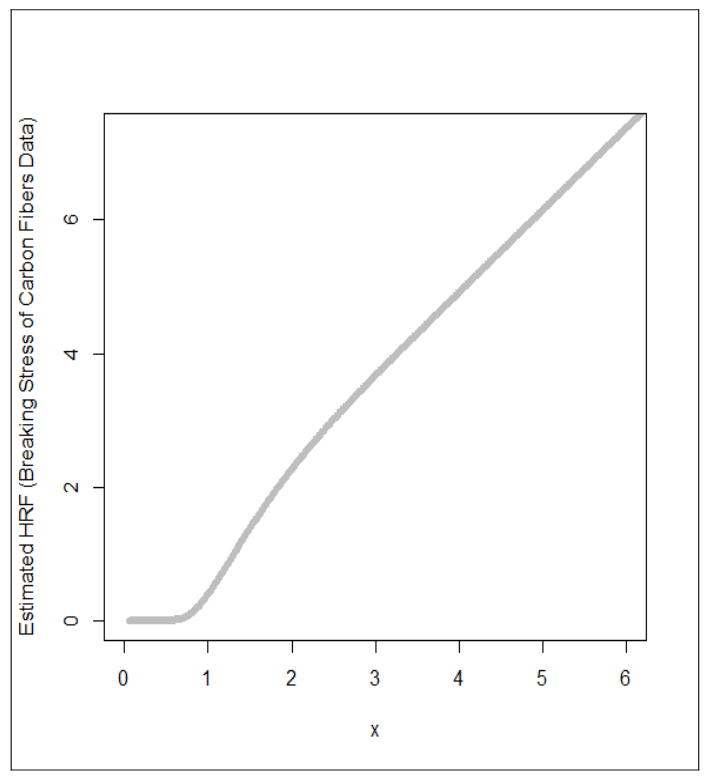

The $1^{\text {st }}$ data.

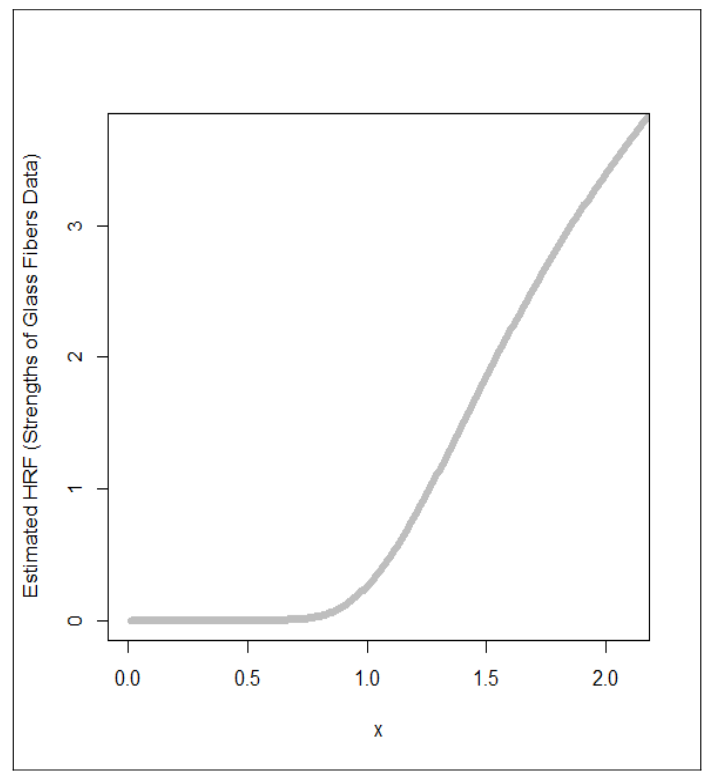

The $2^{\text {nd }}$ data.

Figure 3: Estimated HRFs.

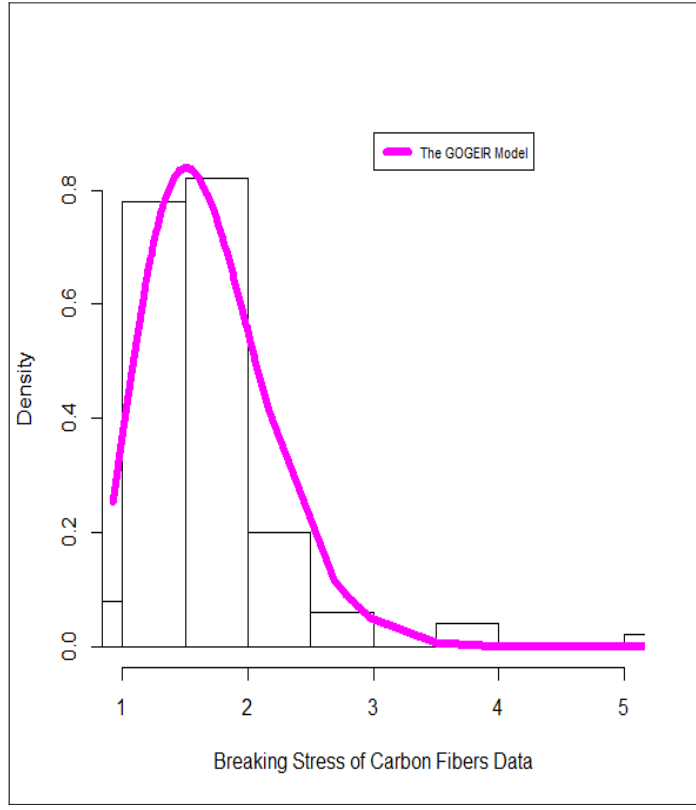

The $1^{\text {st }}$ data.

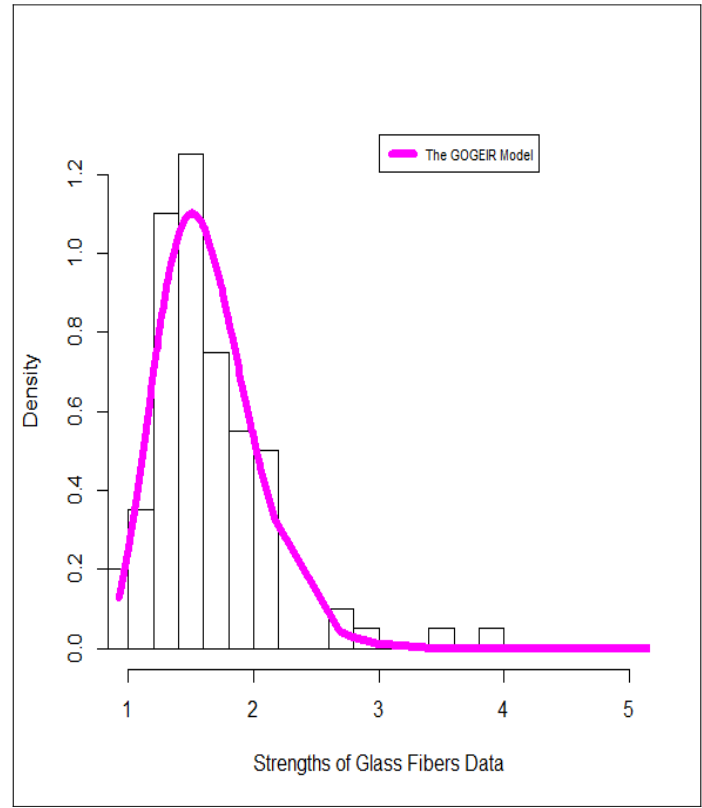

The $2^{\text {nd }}$ data.

Figure 4: Estimated PDFs. 


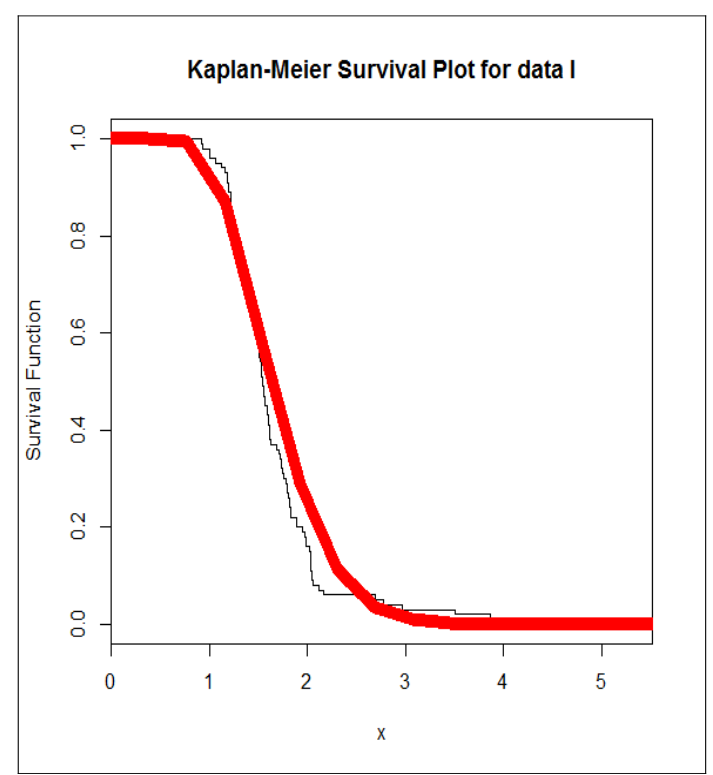

The $1^{\text {st }}$ data.

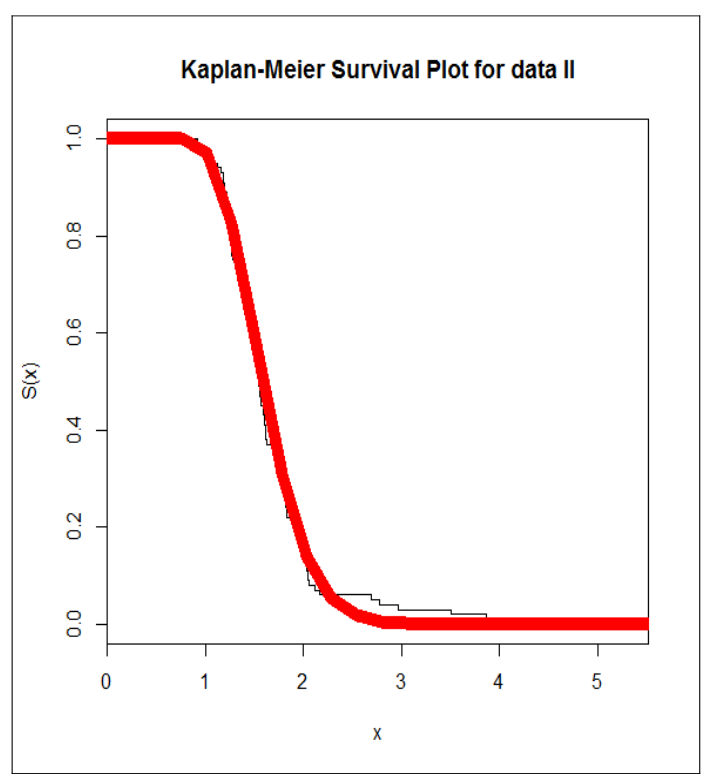

The $2^{\text {nd }}$ data.

Figure 5: Kaplan-Meier Survival Plots.

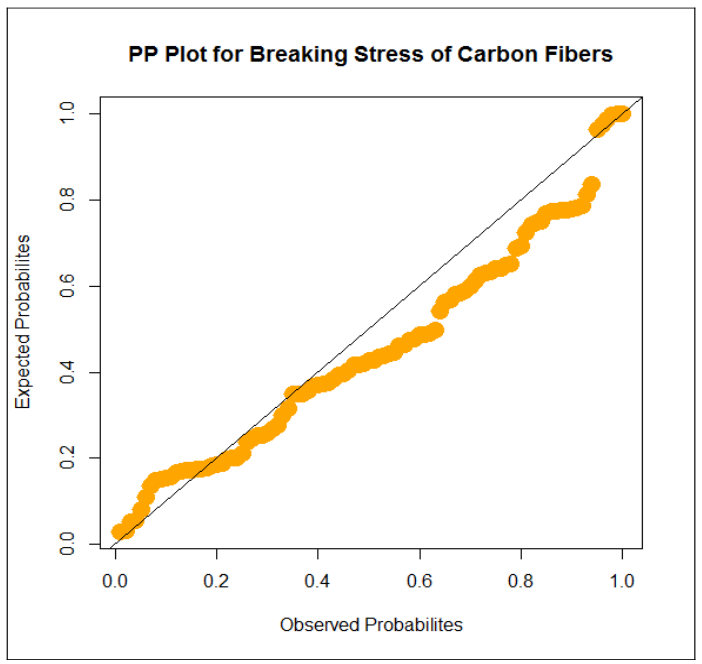

The $1^{\text {st }}$ data.

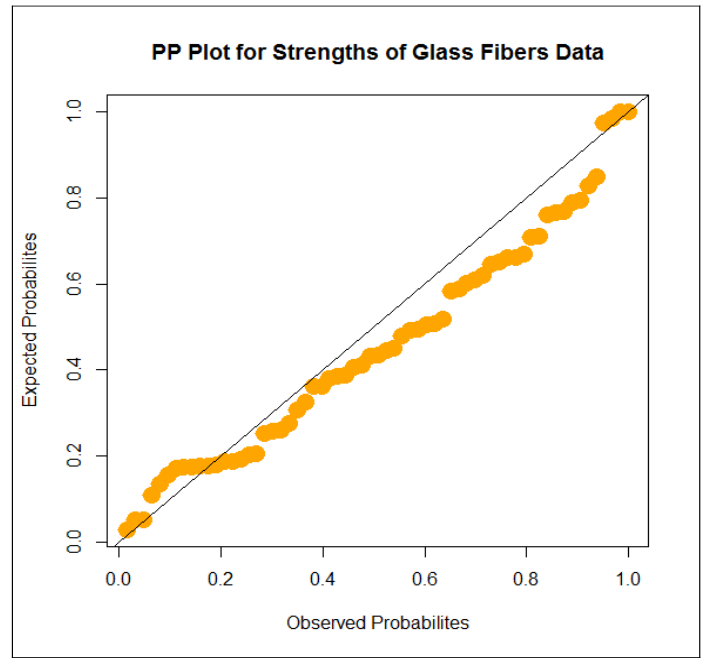

The $2^{\text {nd }}$ data.

Figure 6: P-P plots.

Tables 3 and 5 compare the GOGEIR model with other important competitive distributions. The GOGEIR model gives the lowest values for the AI $c$, BI $c, \mathrm{HQI} c$ and CAI $c$ statistics (in bold values) among all ftted models to these data. So, it may be considered as the best model among them. Figures 2-6, respectively, display the plots of estimated CDFs, estimated PDFs, estimated HRFs, P-P plots and Kaplan-Meier survival plots for the 1 st and 2 nd data. These plots reveal that the proposed distribution yields a sufficient fit for both data sets. 


\section{Conclusions}

A new extension of the Inverse Rayleigh model is proposed and studied. Some of its fundamental statistical properties such as some stochastic properties, ordinary and incomplete moments, moments generating functions, residual life and reversed residual life functions, order statistics, quantile spread ordering, Rényi, Shannon and q-entropies are derived. A simple type Copula based construction via Morgenstern family and via Clayton copula is employed to derive many bivariate and multivariate extensions of the new model. We assessed the performance of the maximum likelihood estimators using a simulation study. The importance of the new model is shown via two applications to real data sets.

\section{References}

1. Abdel-Monem, A. A. 2003. Estimation and Prediction for the Inverse Rayleigh life distribution. M.Sc. Thesis. Faculty of Education, Ain Shames University.

2. Afify, A. Z., Yousof, H. M., Cordeiro, G. M., Ortega, E. M. M. and Nofal, Z. M. (2016). The Weibull Fréchet distribution and its applications. Journal of Applied Statistics, 43(14): 2608-2626.

3. Ahmad, F., Ahmad, S.P. and Ahmed, A. (2014). Transmuted inverse Rayleigh distribution: a generalization of the inverse Rayleigh distribution. Mathematical Theory and Modeling, 4(7): 1-9.

4. Aryal, G. R. and Yousof, H. M. (2017). The exponentiated generalized-G Poisson family of distributions. Economic Quality Control, 32(1): 1-17.

5. Aslam, M. and Jun, C. H. (2009). A group acceptance sampling plans for truncated life tests based on the inverse Rayleigh and log-logistic distributions, Pak. J. Statist., 25(2): 107-119.

6. Barreto-Souza, W. M., Cordeiro, G. M. and Simas, A. B. (2011). Some results for beta Fréchet distribution. Communication in Statistics-Theory and Methods, 40: 798-811.

7. Brito, E., Cordeiro, G. M., Yousof, H. M., Alizadeh, M. and Silva, G. O. (2017). ToppLeone Odd Log-Logistic Family of Distributions, Journal of Statistical Computation and Simulation, 87(15): 3040-3058.

8. Chakraborty, S., Handique, L., Altun, E. and Yousof, H. M. (2018). A new statistical model for extreme values: mathematical properties and applications. International Journal of Open Problems in Computer Science and Mathematics, 12(1): 1-18.

9. Cordeiro, G. M., Yousof, H. M., Ramires, T. G. and Ortega, E. M. M. (2018). The Burr XII system of densities: properties, regression model and applications. Journal of Statistical Computation and Simulation, 88(3): 432-456.

10. Dey, S. (2012). Bayesian estimation of the parameter and reliability function of an Inverse Rayleigh distribution. Malaysian Journal of Mathematical Sciences, 6(1):113-124.

11. Hamedani G. G., Altun, E, Korkmaz, M. C., Yousof, H. M. and Butt, N. S. (2018). A new extended $G$ family of continuous distributions with mathematical properties, characterizations and regression modeling. Pak. J. Stat. Oper. Res., 14 (3): 737-758.

12. Hamedani G. G. Rasekhi, M., Najibi, S. M., Yousof, H. M. and Alizadeh, M., (2019). Type II general exponential class of distributions. Pak. J. Stat. Oper. Res., XV(2): 503523.

13. Hamedani G. G. Yousof, H. M., Rasekhi, M., Alizadeh, M., Najibi, S. M. (2017). Type I general exponential class of distributions. Pak. J. Stat. Oper. Res., XIV(1): 39-55.

14. Korkmaz, M. C., Alizadeh, M., Yousof, H. M. and Butt, N. S. (2018). The generalized odd Weibull generated family of distributions: statistical properties and applications. 
Pak. J. Stat. Oper. Res., 14 (3): 541-556.

15. Korkmaz, M. C., Altun, E., Yousof, H. M. and Hamedani G. G. (2019). The Odd Power Lindley Generator of Probability Distributions: Properties, Characterizations and Regression Modeling, International Journal of Statistics and Probability, 8(2): 70-89.

16. Korkmaz, M. C. Yousof, H. M. and Ali, M. M. (2017). Some Theoretical and Computational Aspects of the Odd Lindley Fréchet Distribution, Journal of Statisticians: Statistics and Actuarial Sciences, 2: 129-140.

17. Kundu, D. and Raqab, M. Z. (2009). Estimation of $\mathrm{R}=\mathrm{P}(\mathrm{X}<\mathrm{Y})$ for three-parameter Weibull distribution. Statistics and Probability Letters, 79: 1839-1846.

18. Mahmoud, M. R. and Mandouh, R. M. (2013). On the transmuted Fréchet distribution. Journal of Applied Sciences Research, 9: 5553-5561.

19. Mukerjee, S.P. and Saran, L.K. (1984). Bivariate inverse Rayleigh distributions in reliability studies. J. Ind. Statist. Assoc., 22: 23-31.

20. Nadarajah, S. and Kotz, S. (2003). The exponentiated exponential distribution. Available online at http://interstat.stat journals.net/YEAR/2003/abstracts/0312001.php

21. Nichols, M. D, Padgett, W. J. (2006). A Bootstrap control chart for Weibull percentiles. Quality and Reliability Engineering International, 22: 141-151.

22. Ramos, M. W. A. Marinho, P. R. D. Cordeiro, G. M., Silva, R. V. da and Hamedani, G. G. (2015) The Kumaraswamy-G Poisson family of distributions, J. Stat. Theory Appl. 14: 222-239.

23. Ristić, M. M. and Nadarajah, S. (2014). A new lifetime distribution, Journal of Statistical Computation and Simulation, 84(1): 135-150

24. Smith, R. L. and Naylor, J. C. (1987). A comparison of maximum likelihood and Bayesian estimators for the three-parameter Weibull distribution. Applied Statistics, 36: 358-369.

25. Soliman, A., Essam A. Amin and Alaa A. Abd-EI Aziz. 2010. Estimation and Prediction from Inverse Rayleigh Distribution Based on Lower Record Values. Applied Mathematical Sciences. 4: 3057-3066.

26. Voda, V. Gh. (1972). On the Inverse Rayleigh Distributed Random Variable. Rep. Statis. App. Res. JUSE. 19(4): 13-21.

27. Yousof, H. M., Afify, A. Z., Alizadeh, M., Butt, N. S., Hamedani, G. G. and Ali, M. M. (2015). The transmuted exponentiated generalized-G family of distributions. Pak. J. Stat. Oper. Res., 11: 441-464.

28. Yousof, H. M., Afify, A. Z., Ebraheim, A. N., Hamedani, G. G. and Butt, N. S. (2016). On six-parameter Fréchet distribution: properties and applications, Pak. J. Stat. Oper. Res., 12: 281-299.

29. Yousof, H. M., Alizadeh, M., Jahanshahiand, S. M. A., Ramires, T. G., Ghosh, I. and Hamedani G. G. (2017). The transmuted Topp-Leone G family of distributions: theory, char-acterizations and applications, Journal of Data Science. 15: 723-740.

30. Yousof, H. M., Altun, E. and Hamedani, G. G. (2018a). A new extension of Frechet distribu-tion with regression models, residual analysis and characterizations. Journal of Data Science, 16(4): 743-770.

31. Yousof, H. M., Jahanshahi, S. M., Ramires, T. G Aryal, G. R. and Hamedani G. G. (2018b). A new distribution for extreme values: regression model, characterizations and applications. Journal of Data Science, 16(4): 677-706. 